\title{
Las Tecnologías de la Información y las Comunicaciones y los Servicios Tecnológicos en las Entidades Públicas del Triángulo del Café en Colombia
}

\author{
Carlos Gómez ${ }^{(1,2)^{\star}}$, Francisco Valencia(1) y Carlos Marulanda ${ }^{(1,2)}$ \\ (1) Universidad Nacional de Colombia, Facultad de Administración, Departamento de Informática y \\ Computación. Campus Palogrande. Calle 27 No. 64-60, Manizales-Colombia \\ (2) Universidad de Caldas, Facultad de Ingeniería, Departamento de Sistemas e Informática, Calle 65 No. \\ 26-10, Manizales-Colombia. Email: ch@ieee.org; cemarulandae@unal.edu.co, carlose@ucaldas.edu.co; \\ fjvalenciad@unal.edu.co.
}

Recibido Oct. 23, 2017; Aceptado Ene. 11, 2018; Versión final Feb. 16, 2018, Publicado Ago. 2018

\section{Resumen}

Se presenta y analiza el estado y alcances de la gestión de las tecnologías de la información (TI) y los servicios tecnológicos en las entidades públicas de las ciudades capitales del triángulo del café de Colombia. Se realizó un trabajo de campo que permitió valorar 39 entidades públicas de esta región. Los hallazgos indican que no hay grandes avances en ninguna de las dos categorías. Adicionalmente, el trabajo y los resultados muestran que la estrategia de las tecnologías de la información y los servicios tecnológicos deben estar alineados y bien coordinados para avanzar en su desarrollo. Con los hallazgos del estudio se recomienda un acompañamiento por parte de las Universidades para mejorar los procesos de adopción para hacer más eficiente y efectivo el uso de tecnologías de la información a a la gestión en el sector público.

Palabras clave: tecnologías de la información; servicios tecnológicos; integración tecnológica, TI

\section{Information and Communication Technologies and Technological Services in the Public Entities of the Coffee Triangle, in Colombia}

\begin{abstract}
The present situation and the scope of information technology in public entities of the capital cities of the coffee triangle of Colombia is presented and analyzed. Fieldwork was carried out to evaluate 39 public entities in this region. The findings indicate that there is no great progress in any of the two categories. Additionally, the work and the results show that information technology strategy and the technological services must be aligned and well-coordinated to advance in the sector development. With the findings of the study it is recommended that universities accompany the public entities to improve the processes of adoption to make more efficient and effective the use of information technology in the management of the public sector.
\end{abstract}

Keywords: information technology; technology services; technological integration; IT 


\section{INTRODUCCIÓN}

El desarrollo de las tecnologías de información y comunicaciones (TI), en el mundo, exige de personas empresas y organizaciones una visión de conjunto y una permanente evaluación de lo que se está haciendo. Tal es el caso de las órganos privados y de las entidades públicas, éstas últimas, con una exigencia mayor, dado su papel con la sociedad. Para el caso del presente estudio, se hace una abordaje evaluativo de las estrategias y los servicios tecnológicos ofrecidos por las entidades públicas del triángulo del café de Colombia, desde una mirada integral, holística y muy acorde con los planteamiento que se han venido haciendo desde gobierno nacional para hacer de la estrategia de TI de Colombia, una realidad en dichas entidades. La gestión de las tecnologías de información y comunicaciones, hacen parte fundamental del proceso de desarrollo organizacional y se ha venido reconociendo como un proceso clave de cualquier organización, llegando a convertirse en el fundamento del éxito en el logro de los objetivos empresariales, y a diferencia del gobierno de $\mathrm{TI}$, se preocupa por las estrategias, programas y proyectos que debe desarrollar de manera inmediata para lograr el aporte que se espera de ellas en el cumplimiento de los objetivos empresariales. Wang et al., (2015), establecen que la gestión de TI está estrechamente embebida en procesos y actividades en toda la empresa, Incluyendo unidades de TI y unidades de negocio. Con el aumento de la integración entre los recursos de TI y las operaciones comerciales, existe la necesidad que todo el personal debe estar bien informado sobre cómo alinear los recursos de TI con los objetivos de negocio. Igualmente, importante es la organización y comprensión del papel de la TI y cómo aprovechar los recursos TI para apoyar las operaciones comerciales y las acciones estratégicas.

La administración de TI juega un papel importante en las planificación, organización, control e integración de las actividades de TI de manera sistemática. Tales actividades son coherentes con las funciones de un subsistema de gestión como propone la teoría de sistemas. Varias actividades pueden ser asociadas con el subsistema de gestión de TI. Diversos recursos de TI necesitan ser asignados de una manera integradora para objetivos comunes. Además, la gestión de TI organiza e integra las actividades de TI hacia el logro del rendimiento y objetivos del negocio. Un objetivo de la gestión de TI es alinear los activos y proyectos de TI con los objetivos de negocio y los requisitos. La gestión de TI ayuda a las organizaciones a orientar el despliegue de los activos de TI para producir los resultados esperados del negocio. El valor de la gestión de $\mathrm{TI}$ radica en su capacidad para implementar eficazmente y supervisar las actividades relacionadas con $\mathrm{TI}$, como la gestión de proyectos, las prácticas de desarrollo de sistemas y la evaluación. Representa la gestión de esfuerzos de planificación, organización, control y dirección de las TI dentro de una organización. La gestión de TI representa la capacidad de alcanzar los objetivos empresariales y responder a los cambios.

La importancia de los activos de TI para las prácticas de administración de TI aumenta cuando la empresa enfrenta un entorno más dinámico. En un entorno cambiante, esperan ver un mayor enfoque en aprovechar los activos de TI para apoyar el procesamiento e interpretar la información relacionada con los cambios, apoyar la toma de decisiones dentro de una empresa. Además, las presiones competitivas del entorno cambiante exigen que las empresas utilicen sus activos de TI Para soportar la transformación de procesos sensibles empresariales. Igualmente, Aguilar et al., (2017), coinciden en que la gestión de la demanda de TI se define como un proceso al que se le asigna capital y recursos humanos limitados necesarios para el beneficio global del negocio y permite mejorar la relación entre TI y negocios. Existen seis mecanismos clave de gestión de la demanda: Planificación, gestión de portafolio, delegación de autoridad, planificación financiera, priorización y valoración.

Berghout y Tan (2013) mencionan una serie de "mejores prácticas" para proyectos TI que coinciden con los elementos de caso de negocio asociados con la gestión de TI, como (1) evitar una estimación de costos pobre; (2) evitar gestión ineficaz de las partes interesadas; (3) evitar riesgos insuficientes administración; y (4) evitar una planificación y calidad inadecuadas garantía. Además, Pedroza y Nieto, (2011), explican que las empresas están en la necesidad de adoptar mejores prácticas con respecto a las TI para optimizar la gestión en sus negocios, esta acción no está limitada al uso de un solo método, probablemente puedan adoptar más de un marco de trabajo de mejores prácticas - o al menos partes de varios - si se desea lograr un conjunto completo y efectivo de lineamientos para la gestión de sus procesos.

\section{OTROS ANTECEDENTES}

Actualmente, la gestión de las tecnologías de información está centrada en el concepto de gestión de servicios de TI, que es la gestión de todas las personas, procesos y tecnología que cooperen para asegurar la calidad de vida de servicios de $\mathrm{TI}$, de acuerdo a los niveles de servicio acordados con el cliente. Se basa en funciones tales como sistemas de gestión, gestión de redes, desarrollo de aplicaciones y en los dominios de proceso tales como la gestión del cambio, gestión de nivel de servicio y gestión de problemas, y cuyos principales representantes en el marco de las mejores prácticas son ITIL (Information Technology Infrastructure Library) y la familia de normas ISO (International Organization for Standardization) / IEC (International Electrotechnical 
Commission) 20000. Precisamente en relación a este grupo de normas, la ISO/IEC 20000-1, norma, certificable para cualquier entidad, establece una serie de requisitos y procesos que deben ser cumplidos para contar con un adecuado sistema de gestión de servicios de tecnologías de información, el cual se resumen en la figura 1, adaptada de Álvarez et al., (2016).

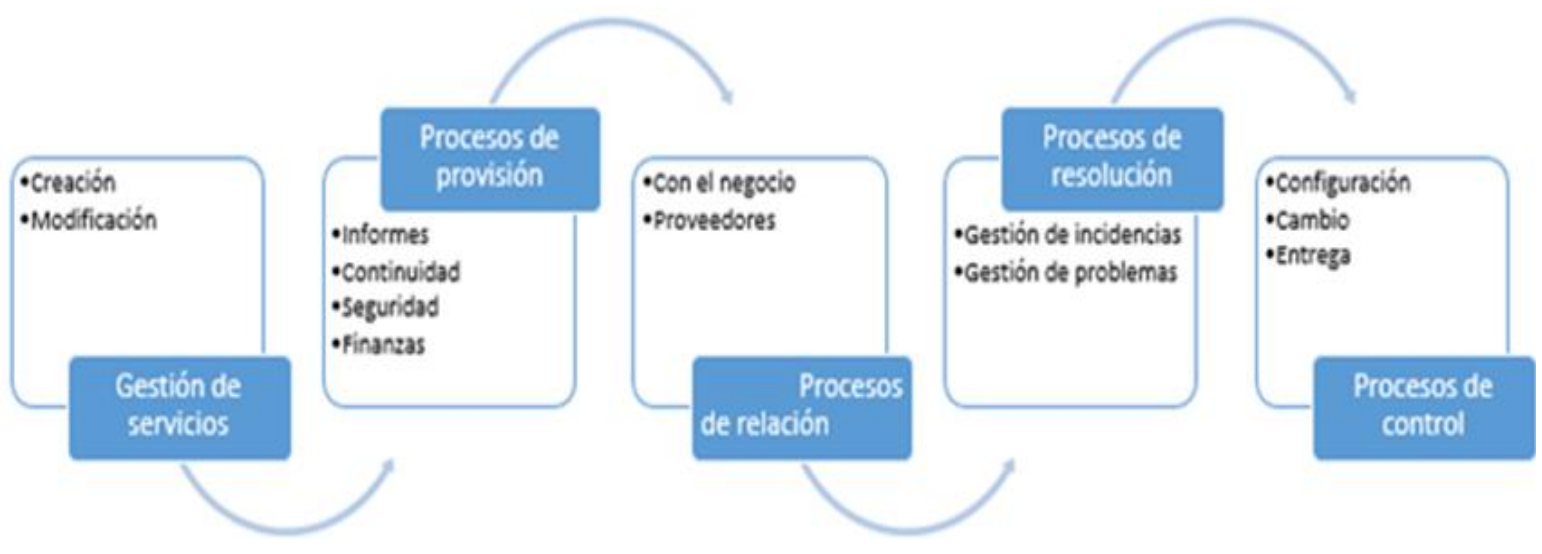

Fig. 1: Sistema de gestión de servicios de TI y sus procesos

En este sentido, Iden y Eikebrokk (2013), explican que cuando se ofrecen servicios de TI deben centrarse en la tecnología y su organización interna y considerar la calidad de los servicios que prestan y la relación con los clientes. La función de TI debe ser ofrecer servicios de TI a un negocio, y la meta es construir y entregar servicios de TI que satisfagan las necesidades y los requisitos de la empresa. La administración de servicios de TI está motivada por: (1) mejorar la eficiencia operativa y reducir el gasto en TI, (2) mejorar el servicio de orientación y concentración en la prestación de servicios, (3) mejorar la alineación, tanto externamente como internamente con los clientes y entre las funciones de TI y (4) mejorar la calidad del servicio y mejorar así la satisfacción del cliente. Por ello la gestión de TI se entiende como el alineamiento estratégico de $\mathrm{TI}$ con la organización de forma tal que se obtenga valor agregado por medio del desarrollo y mantenimiento de un adecuado control y la asignación de responsabilidades, gestión de desempeño y de riesgos. Desde la gestión de TI se busca establecer las estrategias para alcanzar los objetivos institucionales centrado en el uso de las TI y orientado a satisfacer las necesidades.

Es necesario que exista una alineación estratégica de las TI con los objetivos de la administración mediante modelos de gestión aplicados a contextos específicos. Factores políticos, sociales y económicos y estrategias como arquitecturas empresariales, servicios de colaboración, política pública de TI y gobierno electrónico junto a las tendencias tecnológicas hardware y software gestionados adecuadamente pueden proveer un camino más eficaz hacía la construcción de gobernanzas inteligentes, donde la arquitectura empresarial es un elemento fundamental para la gestión por que brinda una visión integral y coherente del gobierno basado en información y tecnología.

En este sentido Rahimi, et. al., (2016), y con una visión más amplia del concepto consideran que el gobierno de $\mathrm{TI}$ es el marco para la distribución de los derechos de toma de decisiones entre las partes interesadas y los procedimientos y mecanismos que forman y supervisan las decisiones de TI. El objetivo de TI es fomentar el comportamiento deseable en el uso de TI y asegurar que la TI de una organización sustente y expanda las estrategias de la organización. Los mecanismos estructurales y de proceso del IT garantizan la definición y el direccionamiento de la estrategia y los principios de TI, la determinación de las prioridades empresariales en las inversiones en TI, la gestión de los riesgos relacionados con las TI y los problemas de seguridad, la gestión de la medición del rendimiento de las TI y la supervisión de la entrega de los beneficios empresariales durante y después de la implementación de los inversores IT.

Al respecto Alreemy et al., (2016), explican que el apoyo de gestión es un paso más después de la participación de los interesados, y su ausencia obstaculizará los próximos pasos de la implementación exitosa de ITG (Gobernanza de tecnologías de información). Además, no se obtendrá el apoyo de ningún directivo de organización ni ejecutivos sin la participación de las partes interesadas. El apoyo debe ser de todos los niveles de gestión para la implementación de ITG, y sin ella, la implementación tendrá dificultades. La gobernanza de TI fue influenciada por la cultura organizacional que resultó ser incompatible con la gestión federal del modelo de gobierno de TI intentada dentro de la organización. Por lo tanto, las empresas deben analizar su cultura y estructura organizacional dentro de la implementación de ITG para asegurar una cultura organizacional de apoyo. Por lo tanto, está claro que el efecto organizacional o interno es un aspecto importante que debe considerarse en la implementación de ITG e incluido en el marco de trabajo. 
En un sentido complementario Martín y Loayza (2015), explican que la gestión de procesos de negocio: o BPM (Business processes management) se posiciona como la primera prioridad para los directivos de TI de las organizaciones estudiadas. El resultado muestra el interés de estas organizaciones en mejorar su eficiencia a través de la gestión integrada y automatizada de sus procesos de negocio. Los sistemas que operan de forma eficiente incrementan el valor del negocio y liberan recursos organizacionales que permiten concentrarse en otros aspectos importantes de la gestión de TI. La organización del área de TI está en una etapa de transición, su rol está pasando de ser tecnológico a uno orientado a los procesos de negocio y a las relaciones con las áreas usuarias. Además (Velásquez et al., 2015), concluyeron que la versión 3 de ITIL, Biblioteca de Infraestructura de Tecnologías de la Información o Information Technology Infrastructure Library en inglés, apoya el alineamiento e integración de la tecnología de la información con la organización, se establece en esta versión un marco de procesos, de roles y las funciones necesarias para una correcta ejecución de TI, está basado en el concepto de ciclo de vida de servicios. ITIL está organizado en 5 fases que van desde la estrategia hasta la mejora continua.

Según Marulanda et. al., (2017), el actual plan de desarrollo de tecnologías de información de Colombia denominado "Vive Digital" para el periodo 2014-2018, liderado por el Ministerio de Tecnologías de Información y Comunicaciones (MINTIC) contempla cuatro líneas estratégicas: empleo, educación, gobierno digital y ciudad región. Dentro de la estrategia de gobierno digital, se contempla como objetivo, tener el gobierno más eficiente y transparente gracias a las TIC, para lo cual se han generado una serie de normas y modelos que apuntan a lograr dicho objetivo, articulados a través de la estrategia de gobierno en línea, cuyos decretos más recientes son los decretos 2573 de 2014, 1078 de 2015 y 415 de 2016. En particular el decreto 1078 establece en su título 9, las políticas y lineamientos de tecnologías de la información para el estado colombiano, a partir del cual se estructuran cuatro componentes de la estrategia de gobierno en línea: TIC para servicios, TIC para el gobierno abierto, TIC para la gestión, y seguridad y privacidad de la información, y establece de forma adicional, el marco de referencia para la gestión de tecnologías de información. Por su parte el decreto 415 de 2016 establece lineamientos para el fortalecimiento institucional en materia de tecnologías de información, donde establece entre otros aspectos la necesidad de considerar la función de TIC en las entidades públicas como una función estratégica para la entidad y establece la obligatoriedad de que esta función haga parte del comité directivo de la entidad, y que dependa de manera directa del representante legal de la misma (artículo 2.2.35.4).

De manera paralela, MINTIC, estableció como modelo de referencia, para el gobierno y gestión de TI en las entidades públicas, el modelo IT4+®, construido a partir de la experiencia, de las mejores prácticas y lecciones aprendidas durante la implementación de la estrategia de gestión TIC en los últimos 10 años. IT4+® es un modelo integral de gestión estratégica con tecnología cuya base fundamental es la alineación entre la gestión de tecnología y la estrategia sectorial o institucional. El modelo facilita el desarrollo de una gestión de TI que genera valor estratégico para el sector, la entidad, sus clientes de información y usuarios. Está conformado por los siguientes componentes: Estrategia de TI, Gobierno de TI, Análisis de información, Sistemas de información, Gestión de servicios tecnológicos, Apropiación y uso. La arquitectura base corresponde a la conceptualización de la arquitectura empresarial para la gestión de las tecnología y sistemas de información del Estado colombiano, en seis dominios o dimensiones: Estrategia, Gobierno, Información, Sistemas de información, Servicios tecnológicos y Uso y apropiación, que será gobernada y alineada a los principios definidos por el Ministerio de las Tecnologías de la Información y las Comunicaciones y que se adoptan implementando el modelo de Gestión IT4+, tal como se puede observar en la figura 2.

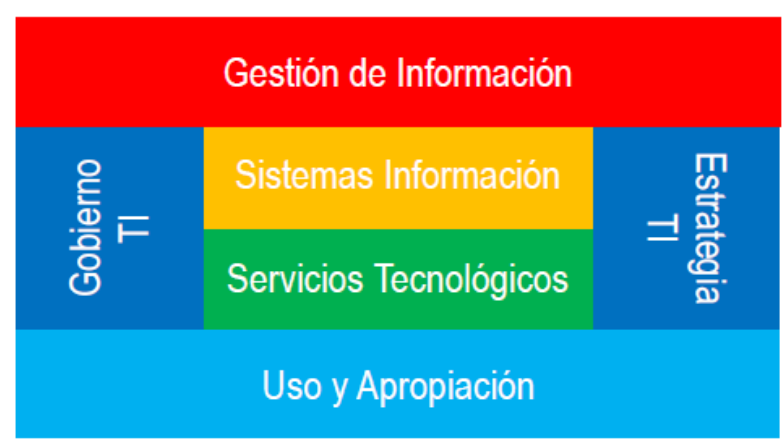

Fig. 2. Modelo IT4+

El modelo busca que la tecnología contribuya al mejoramiento de la gestión apoyando los procesos para alcanzar una mayor eficiencia y transparencia en su ejecución, facilite la administración y el control de los recursos y brinde información objetiva y oportuna para la toma de decisiones en todos los niveles. Permite la alineación de la gestión de TI con los objetivos estratégicos de la entidad, aumentar la eficiencia de la organización y mejorar la forma como se prestan los servicios misionales. 


\section{MATERIALES Y MÉTODOS}

Se desarrolló un tipo de investigación de orden cualitativa, además de tipo de estudio descriptivo, explicativo y correlacional, el cual según Mejía (2004), busca comprender con significados y una perspectiva holística, entender el conjunto de cualidades interrelacionadas que caracterizan a un determinado fenómeno.

\section{Muestreo}

Se aplicó una encuesta a 39 entidades públicas de las ciudades capitales del triángulo del café de Colombia, como son: Manizales (19), Pereira (13) y Armenia (7). Para la estructuración del cuestionario se formularon preguntas en escala Likert, las cuales se calificaron de 1 a 5 , donde 1 , se está en desacuerdo o no realizado, 2 , realizado parcialmente, 3 , realizado en intervalos, 4 , realizado con regularidad y 5 realizado completamente.

\section{Categorías evaluadas}

De acuerdo a las características de valoración, se agruparon las preguntas en las siguientes categorías y variables, (Las categorías y las variables presentadas se valoraron con base en 75 preguntas), tal y como se puede observar en la tabla 1:

Tabla1: Categorías y variables

\begin{tabular}{|l|l|}
\hline \multicolumn{1}{|c|}{ Categorías } & \multicolumn{1}{c|}{ Variables } \\
\hline Estrategia de TI & $\begin{array}{l}\text { Plan de TI, portafolio de planes y proyectos, políticas de TI en cuanto a seguridad, información, } \\
\text { acceso y uso, portafolio de servicios, gestión financiera y plan de Continuidad de TI. }\end{array}$ \\
\hline $\begin{array}{l}\text { Servicios } \\
\text { tecnológicos }\end{array}$ & $\begin{array}{l}\text { Arquitectura de infraestructura tecnológica, Procesos de gestión: capacidad, puesta en producción } \\
\text { y operación, Servicios de conectividad, Servicios de administración y operación, Soporte técnico y } \\
\text { mesa de ayuda y Seguimiento e interventorías }\end{array}$ \\
\hline
\end{tabular}

\section{Análisis de los datos}

Para el análisis de datos Hair et al., (2007), explican la importancia del análisis de correlación, la cual hace posible evaluar simultáneamente varias variables métricas dependientes comparativamente con varias variables métricas independientes. Igualmente, los valores ideales por encima de 0.5.

\section{RESULTADOS Y DISCUSIÓN}

Los resultados que se presentan a continuación se relacionan con la categoría gestión de $\mathrm{TI}$, en las entidades públicas del triángulo del café, que consideran las siguientes variables: Planeación estratégica de gestión de TI (PlanTI), portafolio de planes y proyectos (PortPlan), políticas de TI en cuanto a seguridad, información, acceso y uso (Politic), portafolio de servicios (PortfServ), gestión financiera (GestFina) y plan de Continuidad de TI ( PlanContin). Los resultados se pueden observar en figura 3.

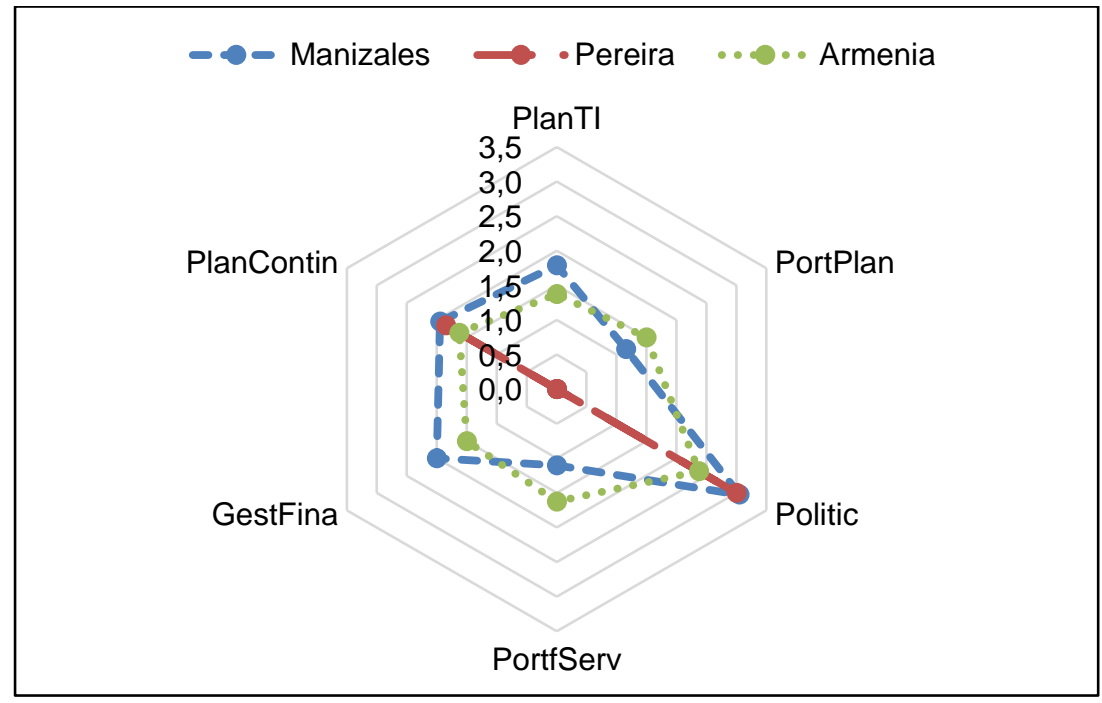

Fig. 3: Estrategia de TI por ciudades 
Las valoraciones observadas muestran como en términos de estrategias de TI, los valores de la tres ciudades en su mayoría están por debajo de 2,0, situación que alarma dad la importancia que debiera tener este importante aspecto organizacional en las entidades evaluadas, mucho más cuando se trata de entidades públicas. En este caso las entidades públicas de la ciudad de Manizales aparecen como las mejor evaluadas y las entidades de la ciudad de Armenia las más bajas. En este marco solo hay una valoración casi igual para las tres ciudades, representada en políticas de TI en cuanto a seguridad, información, acceso y uso, sin embargo esta calificación esta poco más por encima de 3,0. Es preocupante para la comunidad de estas ciudades capitales, que las entidades públicas evaluadas tengan una calificación tan baja, toda vez de la importancia de las TI y sus estrategias para el ejercicio de lo público y la garantía de reglas claras de juego para una sociedad.

En cuanto a la categoría servicios tecnológicos en las entidades públicas del triángulo del café, se consideraron las siguientes: Arquitectura de infraestructura tecnológica (ArqlnfraTec), procesos de gestión: capacidad, puesta en producción y operación (Procgesti), servicios de conectividad (Ssconecti), servicios de administración y operación (SsAdmon), soporte técnico y mesa de ayuda (Soporte) y seguimiento e interventorías (Seglnterve). Los resultados se pueden observar en la figura 4.

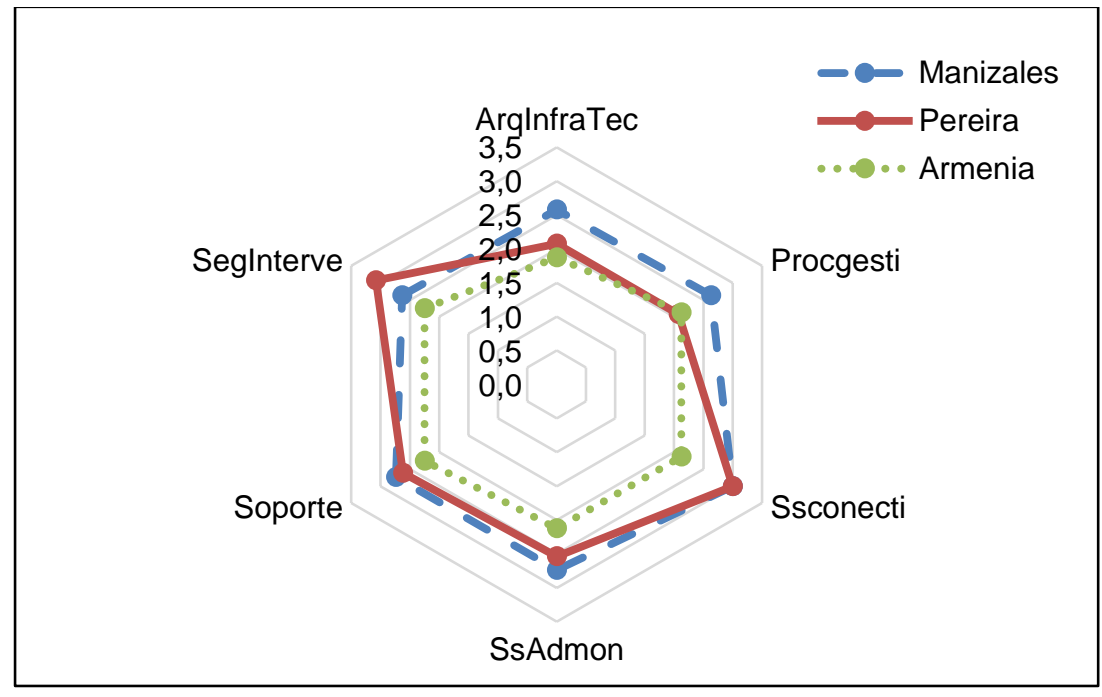

Fig. 4: Servicios tecnológicos por ciudades

La figura presenta que las entidades públicas de la ciudad de Manizales, están calificadas mejor que las demás ciudades en cuanto a: Arquitectura de infraestructura tecnológica, procesos de gestión: capacidad, puesta en producción y operación, servicios de administración y operación y soporte técnico y mesa de ayuda, sin embargo la diferencia es poco significativa, dado que están por debajo de 3,0. Se destacan las entidades de la ciudad de Pereira en seguimiento e interventorías, pero con una calificación apenas por encima de 3,0.

Dichos resultados van en la misma línea de los hallazgos de la categoría de estrategia y se reitera la preocupación de los investigadores en el sentido de unas valoraciones tan bajas para un tema tan importante como los servicios tecnológicos de las entidades públicas del triángulo del café. Dados estos resultados se realizó un análisis de correlación, para determinar la influencia, asociación o relación entre las categorías medidas y los resultados encontrados se pueden observar en la tabla 2.

Los resultados de la correlación muestran que existe una relación directa y significativa entre las categorías y las variables, ya que se cuenta con una cifra mayor al 50\%. Sin embargo preocupa como no se da correlación entre procesos de gestión: capacidad, puesta en producción y operación y servicios de administración y operación con portafolio de planes y proyectos, políticas de TI en cuanto a seguridad, información, acceso y uso, portafolio de servicios y gestión financiera, lo que conduce a explicar que puede existir falta de comunicación o diferencias de criterios o falta de capacitación entre tantas posibilidades, entre quienes plantean las estrategias y quienes hacen realidad los servicio de TI en las entidades públicas del triángulo del café.

Tabla 2: Análisis de correlación de variables. ${ }^{* *}$. La correlación es significativa en el nivel 0,01 (2 colas).

\begin{tabular}{|l|r|l|l|l|l|l|l|l|l|l|l|l|}
\hline & Plan & Portpla & IPol & Portserv & Finan & Plancon & Arq & Gest & Conec & Adm & Sop & Seg \\
\hline \multirow{2}{*}{ Plan } & 1 & \multicolumn{10}{|l}{} \\
\hline
\end{tabular}


Tabla 2 (continuación)

\begin{tabular}{|c|c|c|c|c|c|c|c|c|c|c|c|c|}
\hline & Plan & Portpla & \multirow[t]{2}{*}{ IPol } & \multirow[t]{2}{*}{ Portserv } & \multirow{2}{*}{ Finan } & \multirow{2}{*}{ Plancon } & \multirow{2}{*}{ Arq } & \multirow{2}{*}{ Gest } & \multirow{2}{*}{ Conec } & \multirow{2}{*}{ Adm } & \multirow{2}{*}{ Sop } & \\
\hline Portpla &, $748^{* *}$ & 1 & & & & & & & & & & \\
\hline IPol & ,313 & ,299 & 1 & & & & & & & & & \\
\hline Portserv &, $702^{* \star}$ &, $927^{* \star}$ &, $345^{*}$ & 1 & & & & & & & & \\
\hline Finan &, $679^{* \star}$ &, $705^{\star \star}$ &, $351^{*}$ &, $749^{* *}$ & 1 & & & & & & & \\
\hline Plancon &, $372^{*}$ &, $339^{*}$ &, $454^{* *}$ & ,329* & ,224 & 1 & & & & & & \\
\hline Arq &, $511^{\star \star}$ &, $453^{\star \star}$ &, $398^{*}$ &, $450^{* \star}$ &, $423^{\star \star}$ &, $769^{* *}$ & 1 & & & & & \\
\hline Gest &, $489^{* \star}$ &, $449^{* *}$ &, $353^{*}$ &, $447^{* \star}$ &, $360^{*}$ &, $648^{* *}$ &, $895^{* \star}$ & 1 & & & & \\
\hline Conec & ,334* &, $341^{*}$ &, $526^{* *}$ & ,370* &, $496^{* *}$ &, $519^{* *}$ &, $748^{* *}$ &, $696^{* *}$ & 1 & & & \\
\hline $\mathrm{Adm}$ &, $463^{* \star}$ & $394^{*}$ &, $389^{*}$ &, $404^{*}$ & $370^{*}$ &, $606^{* *}$ &, $871^{* *}$ &, $847^{\star *}$ &, $818^{* *}$ & 1 & & \\
\hline Sop &, $350^{*}$ &, $343^{*}$ &, $585^{* *}$ &, $362^{*}$ &, $391^{*}$ &, $531^{\star *}$ &, $630^{\star *}$ &, $609^{* *}$ &, $872^{* *}$ &, $709^{* \star}$ & 1 & \\
\hline Seg & ,279 &, $331^{*}$ &, $414^{* *}$ & $351^{*}$ &, $447^{* \star}$ &, $506^{* *}$ &, $637^{\star *}$ &, $591^{* *}$ &, $873^{* *}$ &, $695^{* *}$ &, $828^{* *}$ & 1 \\
\hline
\end{tabular}

\section{CONCLUSIONES}

De los resultados presentados, de su análisis y de la discusión, se obtienen las siguientes conclusiones principales:

i) Para el desarrollo de un adecuado sistema de gestión de servicios de tecnologías de información, la definición de estrategias de TI es un componente fundamental, si se quiere hacer de la tecnología un actor estratégico para el desarrollo corporativo, y para el caso de las entidades públicas del triángulo del café, se debería convertir en la base del desarrollo para el mejoramiento de las comunidades que los organismos gubernamentales representan.

ii) Las estrategias y los servicios tecnológicos deben ir de la mano en su desarrollo, procurando la satisfacción del usuario, el ciudadano, que requiere de diversos servicios por parte de las entidades públicas y se nota en los hallazgos que hay diferencias importantes en la integración de estos temas.

iii) Los hallazgos muestran que no existen grandes avances en ninguna de las dos categorías, dada las valoraciones mínimas en ambas, lo que hace necesario dar cumplimiento de forma más asertiva no solo a los lineamientos normativos establecidos por el gobierno colombiano, sino a las mejores prácticas establecidas en marcos de referencia como ITIL y/o la familia de normas ISO/IEC 20000.

iv) Algunas de las causas que pueden estar originando esta falta de correlación entre los servicios de TI y las estrategias tecnológicas que se formulan, de acuerdo al análisis realizado por los autores, es la falta de capacitación y de acompañamiento en la implementación de este tipo de prácticas por parte del Ministerio de Tecnologías de Información.

\section{AGRADECIMIENTOS}

A la Dirección de Investigaciones de la Universidad Nacional sede Manizales DIMA. A la Vicerrectoría de Investigaciones de la Universidad de Caldas. Nombre de la convocatoria: Convocatoria de investigación conjunta Universidad Nacional de Colombia - Universidad de Caldas. Proyecto de Investigación titulado: Análisis del gobierno y gestión de TIC en las entidades públicas del eje cafetero, frente a las directrices de la estrategia de gobierno en línea del Estado Colombiano. Código Hermes: 19328.

\section{REFERENCIAS}

Aguilar, I., J. Carrillo y E. Tovar, Description of the structure of the IT demand management process framework, International Journal of Information Management, (37), 1461-1473 (2017)

Alreemy, Z., V. Chang, R. Walters y G. Wills, Critical success factors (CSFs) for information technology governance (ITG), International Journal of Information Management, (36), 907-916 (2016)

Álvarez, A. A., C. M. Fernández Sánchez y B. Delgado Riss, Guía práctica de ISO/IEC 20000-1 para servicios TIC. Madrid, España, AENOR (2016)

Berghout, E. y C.W. Tan, Understanding the impact of business cases on IT investment decisions: An analysis of municipal e-government projects, Information \& Management (50), 489-506 (2013) 
Ebner, K., N. Urbach y B. Mueller, Exploring the path to success: A review of the strategic IT benchmarking literature, Information \& Management, (53), 447-466 (2016)

Iden, J., y T. Eikebrokk, Implementing IT Service Management: A systematic literature review. International Journal of Information Management, (33), 512- 523 (2013)

Maestre, G. y W. Nieto, Factores Clave en la Gestión de Tecnología de Información para Sistemas de Gobierno Inteligente, Journal of Technology Management \& Innovation, 10(4), 95-104 (2015)

Martín, S. y A. Loayza, Prioridades de gestión de tecnologías de información en organizaciones peruanas, Revista Venezolana de Gerencia, (72), 684-697 (2015)

Marulanda, C., F. Valencia y M. López, Gobierno y gestión de TI en las entidades públicas de Manizales, Colombia. Administer, 1-21 (2017)

Mejía, N.J., Sobre la investigación cualitativa, Investigaciones sociales, 2ª Ed., McGraw Hill, Madrid (2004)

Pedroza, P. y W. Nieto, Modelo para la gestión de la calidad del servicio de TI aplicable a las pymes de la ciudad de Barranquilla, Revista Generación Digital, 9(1), 1-9 (2011)

Rahimi, F., C. Møller y L. Hvam, Business process management and IT management: The missing integration, International Journal of Information Management, (36), 142-154 (2016)

Velásquez, T., A. Puentes e Y. Pérez, Un enfoque de buenas prácticas de gobierno corporativo de TI, Revista Tecnura (19), 159-169 (2015)

Wang, Y., S. Shi, S. Nevo, S. Li, S. e Y. Chen, The interaction effect of IT assets and IT management on firm performance: A systems perspective, International Journal of Information Management, (35), 580-593 (2015) 